\title{
ORIGINAL ARTICLE KLF4 functions as an activator of the androgen receptor through reciprocal feedback
}

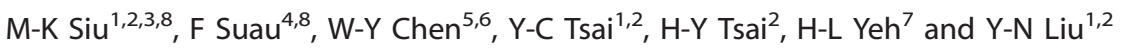

In prostate cancer, Krüppel-like factor 4 (KLF4) depletion occurs frequently, suggesting a role as suppressor tumor. KLF4 is a transcription factor associated with androgen receptor (AR) expression; however, its cellular functions and signaling regulation mechanism remain largely unknown. In this study, we demonstrated that activated AR binds to the KLF4 promoter and enhances KLF4 expression, which reciprocally targets the $A R$ promoter, thus sustaining KLF4 activity. Ectopic KLF4 expression in androgenindependent prostate cancer cells induced AR expression and decreased cell proliferation, invasion and bone metastasis. We previously showed that increased microRNA (miR)-1 expression is associated with reduced bone metastasis of prostate cancer cells. Here we observed that KLF4 targets the primary miR-1-2 stem-loop promoter and stimulates miR-1 expression. In clinical prostate cancer specimens, KLF4 levels were positively correlated with miR-1 and AR levels. These data suggest that the loss of KLF4 expression is one mechanistic link between aggressive prostate cancer progression and low canonical AR output through miR-1 inactivation.

Oncogenesis (2016) 5, e282; doi:10.1038/oncsis.2016.79; published online 19 December 2016

\section{INTRODUCTINON}

Kruppel-like factor 4 (KLF4), which belongs to the KLF family of transcription factors, regulates diverse cellular functions depending on tissue and tumor type or cancer stage and may have a role as a tumor suppressor or oncogene. ${ }^{1}$ KLF4 inhibits tumor progression in prostate cancer and that the loss of KLF4 expression is a diagnostic marker in patients with advanced prostate cancer, ${ }^{2,3}$ consistent with the role of KLF4 in cell cycle arrest and growth inhibition. ${ }^{1,4,5}$ Moreover, the loss of one allele of KLF4 can induce intestinal adenoma development in $\mathrm{Apc}^{\mathrm{Min}}$ mice, which corroborates the tumor-suppressive effect of this protein. ${ }^{6}$ Notably, KLF4 expression is significantly correlated with activated androgen receptor (AR) signaling in prostatic stromal cells ${ }^{7}$ and circulating tumor cells of prostate cancer patients, ${ }^{8}$ suggesting an association between KLF4 and AR in prostate cancer cells.

$A R$ is activated after the binding of androgenic ligands and has a pivotal role in prostate cancer development. ${ }^{9,10}$ AR has become the most crucial therapeutic target in prostate cancer treatment. ${ }^{11-13}$ However, many treatments targeting AR signaling are noncurative and associated with a poor prognosis, despite continuous hormonal manipulation. Therefore, the resistance mechanisms and molecular pathways involved in changes of AR function as prostate cancer progresses are being increasingly investigated.

The microRNAs (miRs) represent a class of small, regulatory, noncoding RNA molecules that can become dysregulated in cancer. ${ }^{14}$ Altered miR expression and the subsequent effects on target gene transcription have been frequently associated with metastatic trait acquisition. ${ }^{15}$ miR-1 has been proposed as a candidate tumor suppressor and prognostic marker for prostate cancer. ${ }^{16-18}$ We previously established that ectopic miR-1 expression negatively affects the growth ability of prostate cancer cells. ${ }^{19}$ Notably, we demonstrated that miR-1 expression suppresses experimental bone metastasis and that miR-1 is directly and positively modulated by AR signaling. ${ }^{20}$

In this study, we explored the regulatory mechanisms linking AR signaling with miR-1 expression in prostate cancer. We demonstrated that KLF4 expression is directly and transcriptionally upregulated by $A R$, and AR expression is reciprocally upregulated by KLF4. Moreover, in prostate cancer cell lines, KLF4 binds to the miR-1 promoter and induces its expression. Our findings also revealed that KLF4 is positively associated with AR and miR-1 levels in patient tissue samples. Here, we established a central role for KLF4 in tumor suppression by connecting its regulation by AR and modulation of miR-1 expression.

\section{RESULTS}

Induction of KLF4 expression is associated with activated AR signaling

Although KLF4 activity is repressed in several cancers and can have a tumor-suppressive effect, ${ }^{2,5,21}$ the specific role of KLF4 in AR pathway-activated prostate cancer remains unclear. We analyzed the relationship between KLF4 and AR signaling and observed that AR-expressing cells LNCaP, LNCaP-AR and 22Rv1 had higher KLF4 expression than did cells without AR expression PC3, DU145 and RasB1 (Figure 1a). In LNCaP and LNCaP-AR cells,

\footnotetext{
${ }^{1}$ Program for Cancer Biology and Drug Discovery, College of Medical Science and Technology, Taipei Medical University and Academia Sinica, Taipei, Taiwan; ${ }^{2}$ Graduate Institute of Cancer Biology and Drug Discovery, College of Medical Science and Technology, Taipei Medical University, Taipei, Taiwan; ${ }^{3}$ Department of Anesthesiology, Wan Fang Hospital, Taipei Medical University, Taipei, Taiwan; ${ }^{4}$ Department of Microbiology, Faculty of Pharmacy, Dicle University, Diyarbakir, Turkey; ${ }^{5}$ Department of Pathology, Wan Fang Hospital, Taipei Medical University, Taipei, Taiwan; ${ }^{6}$ Department of Pathology, School of Medicine, College of Medicine, Taipei Medical University, Taipei, Taiwan and ${ }^{7}$ Institute of

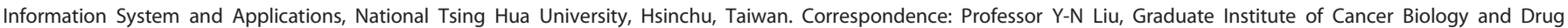
Discovery, College of Medical Science and Technology, Taipei Medical University, 250 Wu-Hsing Street, Taipei 11031, Taiwan.

E-mail: liuy@tmu.edu.tw

${ }^{8}$ These two authors contributed equally to this work.

Received 19 July 2016; revised 7 October 2016; accepted 2 November 2016
} 
a

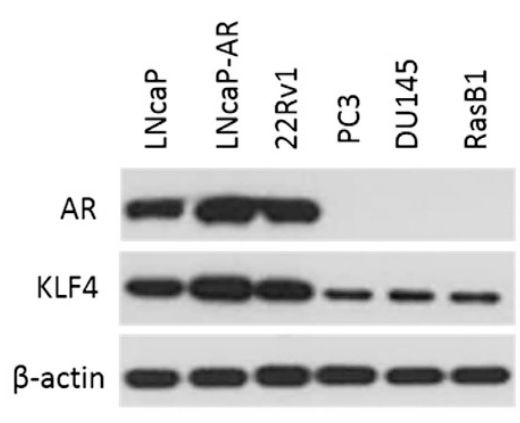

C

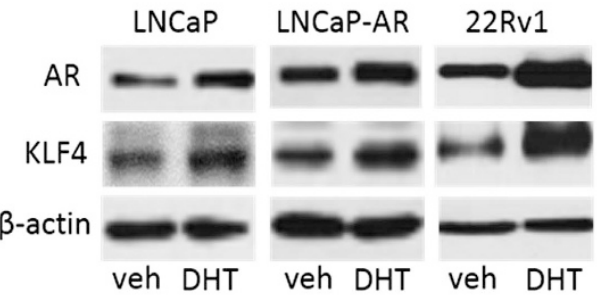

e

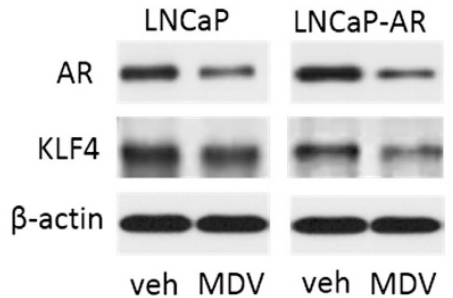

g

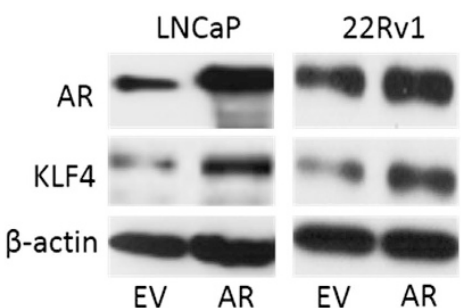

b

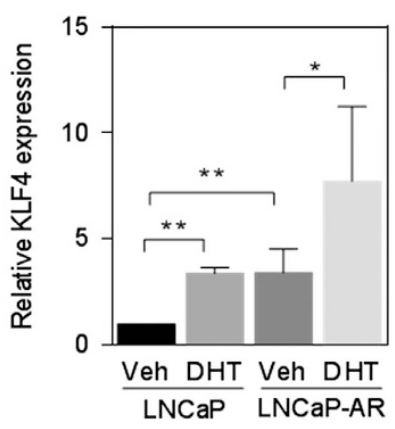

d

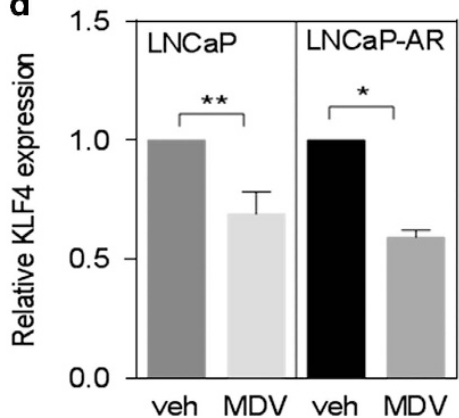

f

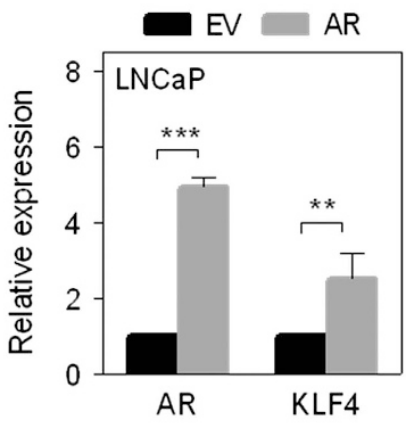

h

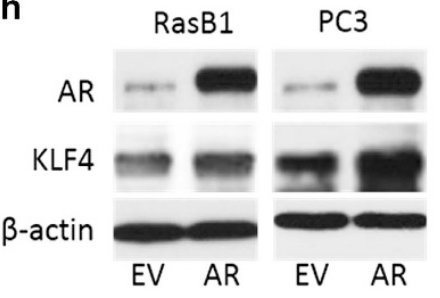

Figure 1. Activated androgen receptor (AR) signaling induces Kruppel-like factor 4 (KLF4) expression. (a) Immunoblots for detecting endogenous AR and KLF4 levels in various prostate cancer cell lines. (b) KLF4 mRNA levels in LNCaP and LNCaP-AR cells following DHT treatment (10 $\mathrm{nm}$ for $24 \mathrm{~h}$ in a $10 \%$ charcoal-stripped FBS-containing medium). (c) Immunoblots for detecting AR and KLF4 levels in LNCaP, LNCaP-AR and 22Rv1 cells following DHT treatment. (d) KLF4 mRNA levels in LNCaP and LNCaP-AR cells following MDV3100 treatment (10 $\mu \mathrm{M}$ for $24 \mathrm{~h}$ in a $10 \%$ FBS-containing medium). (e) Immunoblots for detecting AR and KLF4 levels in LNCaP and LNCaP-AR cells following MDV3100 treatment. (f) Quantitative real-time reverse-transcription PCR analysis of AR and KLF4 in LNCaP cells after ectopic AR or empty vector (EV) expression. (g) Representative immunoblot analysis of AR and KLF4 protein expression in LNCaP and 22 Rv1 cells following EV or AR expression. (h) Immunoblots for detecting AR and KLF4 protein expression in RasB1 and PC3 cells following EV or AR expression. All the experiments were performed in triplicate, and the data are presented as the mean \pm s.e.m., $n=3$. ${ }^{*} P<0.05,{ }^{* *} P<0.01$, ${ }^{* * *} P<0.001$.

KLF4 messenger RNA (mRNA) and protein levels increased after dihydrotestosterone (DHT) treatment (Figures $1 \mathrm{~b}$ and $\mathrm{c}$ ), but decreased after treatment with MDV3100, an AR antagonist ${ }^{22,23}$ (Figures 1d and e), suggesting an association between KLF4 and AR signaling in the same cell signaling pathway in AR-positive prostate cancer cells. Moreover, KLF4 mRNA levels increased in LNCaP cells transfected with an AR expression vector (Figure 1f).
The increase in KLF4 levels was confirmed by immunoblotting extracts from LNCaP and 22Rv1 cells, demonstrating that KLF4 expression increases in the presence of AR expression (Figure 1g). These results were further confirmed by immunoblotting extracts from AR-negative RasB1 and PC3 cells ectopically overexpressing AR (Figure 1h). Taken together, these data suggest that KLF4 expression is upregulated by $A R$ in prostate cancer. 
a

Human KLF4 promoter (GRCh37:9)

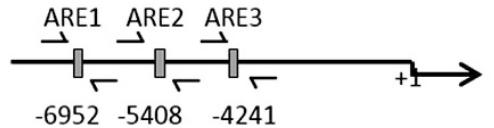

Wild type Mutant

ARE1 $\rightarrow$ REP AGAACA $\rightarrow$ GAATTC

ARE2 - REP TGTTCT $\rightarrow$ GAATTC

ARE3 $\rightarrow$ REP TGTTCT $\rightarrow$ GAATTC

C
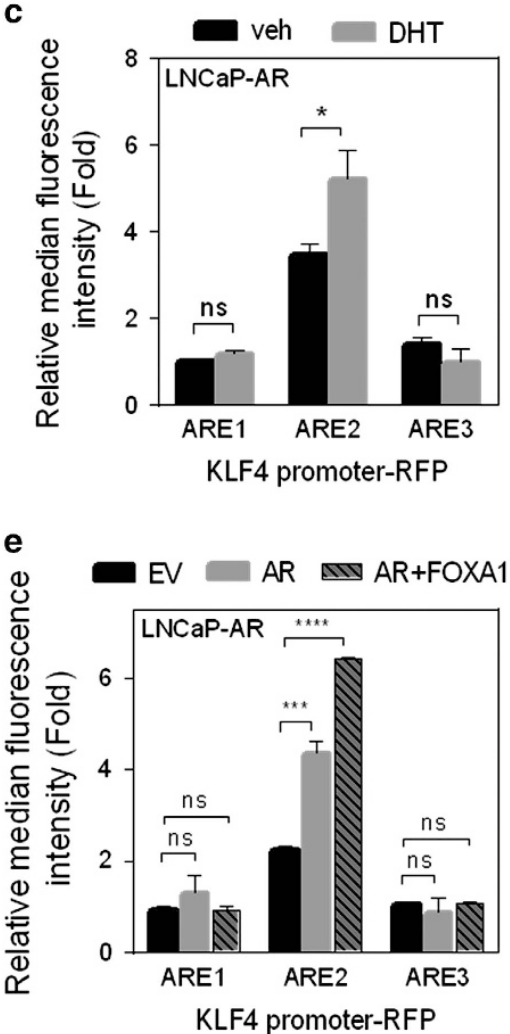

b
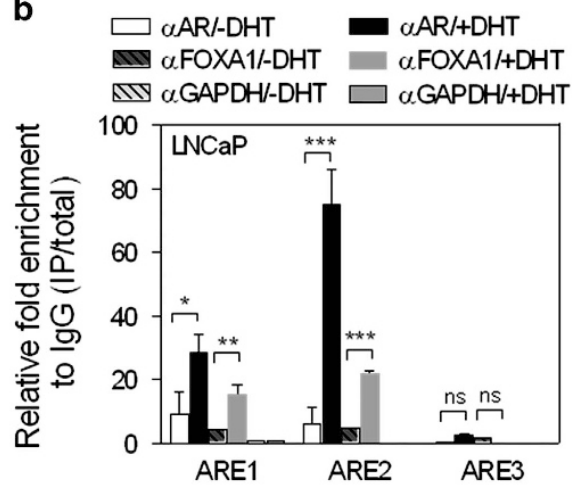

d

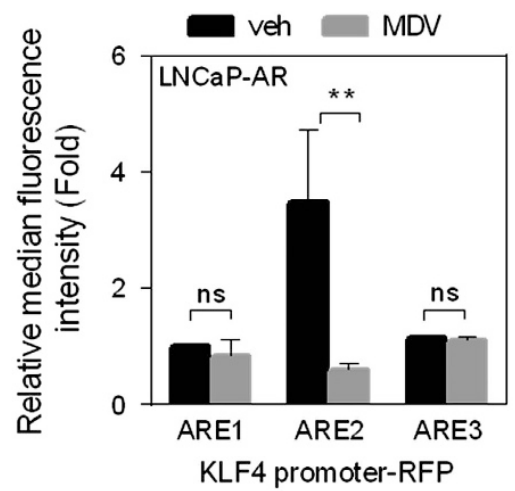

f

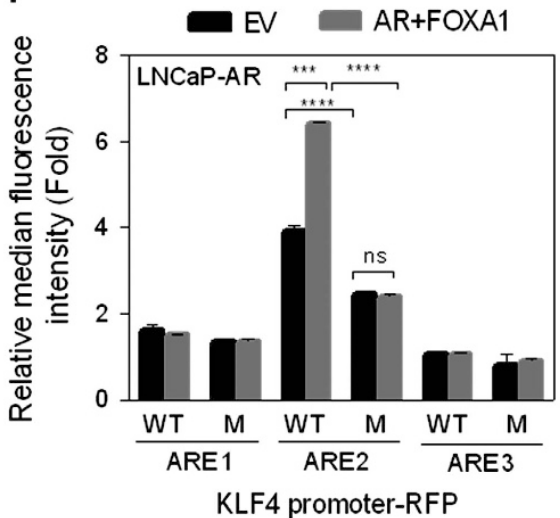

Figure 2. Activated androgen receptor (AR) stimulates Kruppel-like factor 4 (KLF4) expression by enhancing the KLF4 promoter. (a) Schematic of the predicted AR responsive elements (AREs) and an introduced binding site mutant in promoter reporter constructs of the human KLF4 promoter at $-6952,-5408$ and -4241 bp upstream. (b) Chromatin immunoprecipitation assays for AR and FOXA 1 binding to predicted AREs in the KLF4 promoter region measured in LNCaP cells treated with $10 \mathrm{~nm}$ DHT for $4 \mathrm{~h}$. The binding activity of each protein to each site is given as a percentage of the total input, normalized to each IgG. (c and d) Activity of a RFP reporter gene containing AREs from the KLF4 promoter. Expression of the transiently transfected reporter gene, normalized to gene expression from a transfected control vector, was assayed in LNCaP-AR cells following $24 \mathrm{~h}$ treatment with $10 \mathrm{~nm} \mathrm{DHT} \mathrm{(c)} \mathrm{or} 10 \mu \mathrm{m}$ MDV3100 (d). Relative median fluorescence intensity (MFI) of the reporter protein following DHT or MDV3100 treatment. (e) Activity of the same RFP reporter gene as in c when LNCaP-AR cells were transfected with a plasmid expressing AR, FOXA1, or a control empty vector (EV). The MFI normalized to a control for transfection efficiency is shown. (f) The LNCaP-AR cells were transiently cotransfected with wild-type (WT) or ARE-mutated ARE-RFP reporters with AR or FOXA1 expression vectors for $48 \mathrm{~h}$. The MFI was measured through fluorescence-activated cell sorting and normalized to the value of the EV. All the experiments were performed in triplicate and the data are presented as the mean \pm s.e.m., $n=3$. NS, nonsignificant, ${ }^{*} P<0.05,{ }^{* *} P<0.01,{ }^{* *} P<0.001$, $* * * * P<0.0001$.

AR binding to the KLF4 promoter directly and positively regulates KLF4 expression

We next investigated whether AR stimulates KLF4 expression through binding and transcriptional regulation of the KLF4 promoter. We evaluated the KLF4 promoter for the presence of potential AR-binding sites and identified three putative AR homologous responsive elements (AREs) in the upstream region of the KLF4 promoter (Figure 2a). FOXA1, an AR cofactor, can cooperate with $\mathrm{AR}$ to mediate gene expression in prostate cancer. $^{24-26}$ To determine whether AR and FOXA1 effectively bind to the KLF4 promoter, we performed quantitative reversetranscription PCR (qRT-PCR) analysis in LNCaP cells after a chromatin immunoprecipitation (ChIP) assay by using anti-AR and anti-FOXA1 antibodies. The binding of AR and FOXA1 was 
observed only at the ARE1 and ARE2 motifs, and the binding significantly increased after DHT treatment (Figure 2b). Next, to assess the effect of AR on the transcriptional activity of $K L F 4$, the LNCaP-AR cells were transfected with RFP reporter constructs containing the individual predicted AREs of the KLF4 promoter. DHT treatment of LNCaP-AR cells significantly increased ARE2 reporter activity but not that of ARE1 or ARE3 (Figure 2c); in addition, we noted concordantly decreased reporter activity after treatment of the same cells with MDV3100 (Figure 2d). Furthermore, ARE2 reporter activity increased in LNCaP-AR cells transfected with AR compared with cells transfected with a control vector, and the activity increased further in cells a

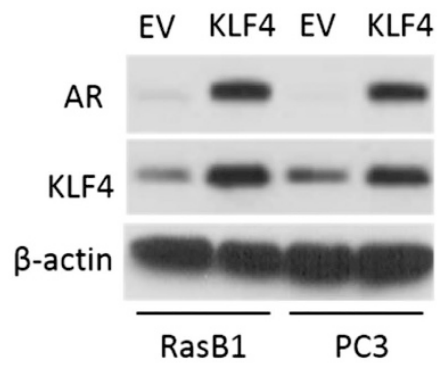

C

Human $A R$ promoter (GRCh38: X)

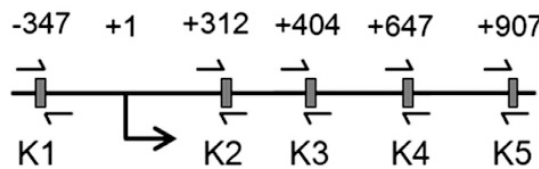

KLF4 RE Wild type Mutant

$\mathrm{K} 1-\mathrm{RFP}$ CACCCT $\rightarrow$ GGTACC

$\mathrm{K} 4-\mathrm{RFP}$ CACCCC $\rightarrow$ GGTACC

e

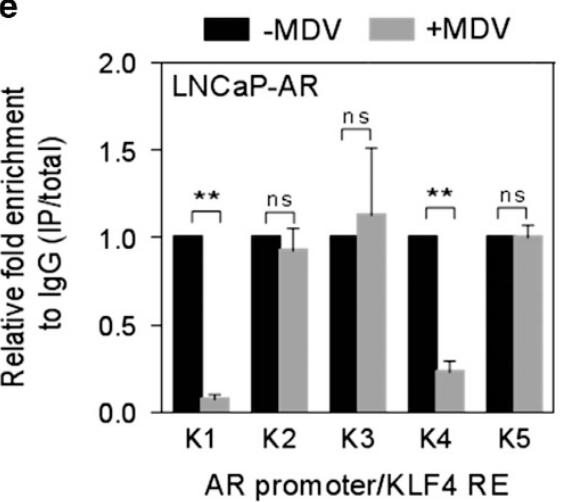

g

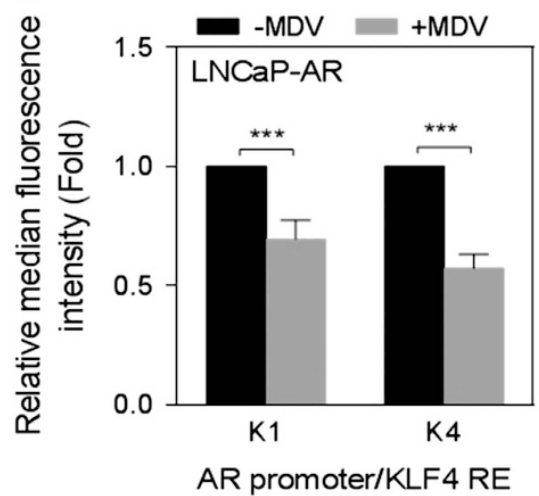

b

shRNA Luc KLF4 Luc KLF4

AR

KLF4

ß-actin

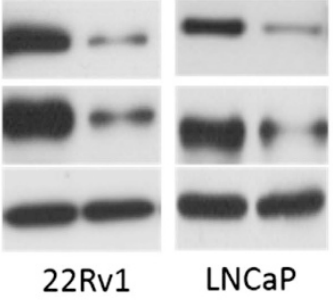

d

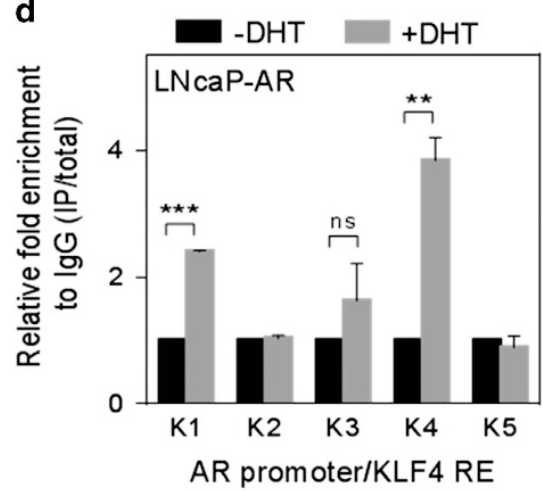

f

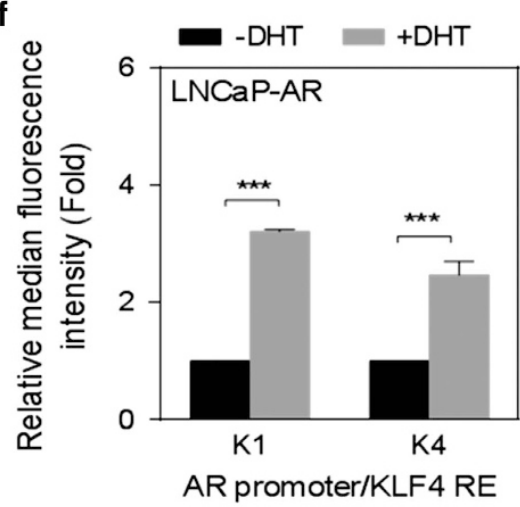

h

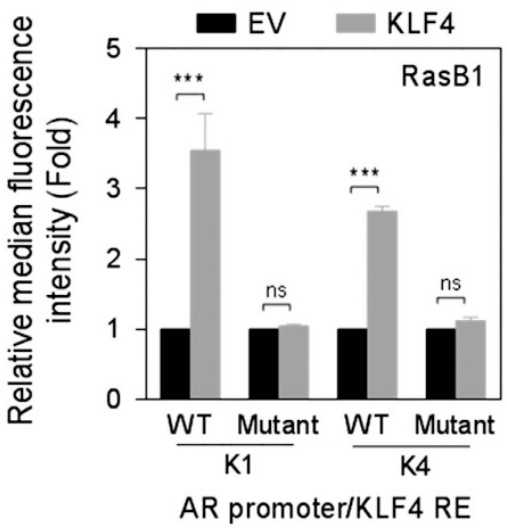




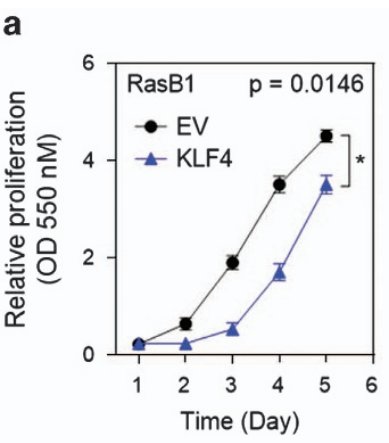

d

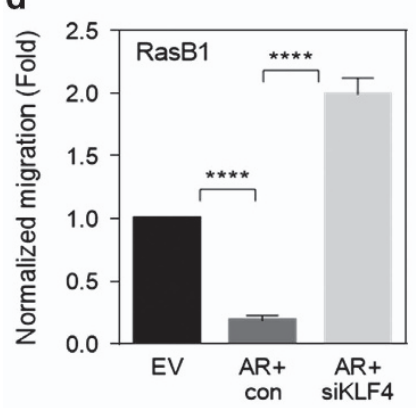

g

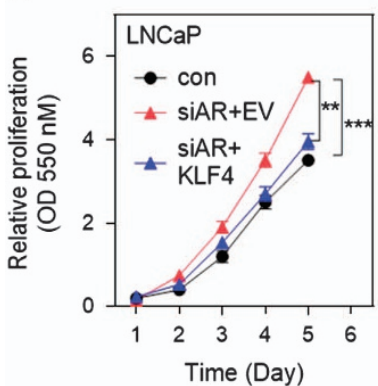

b

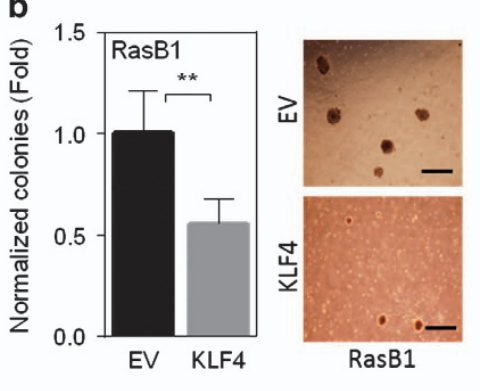

e

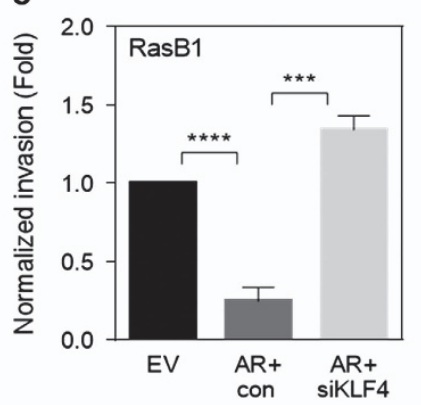

C

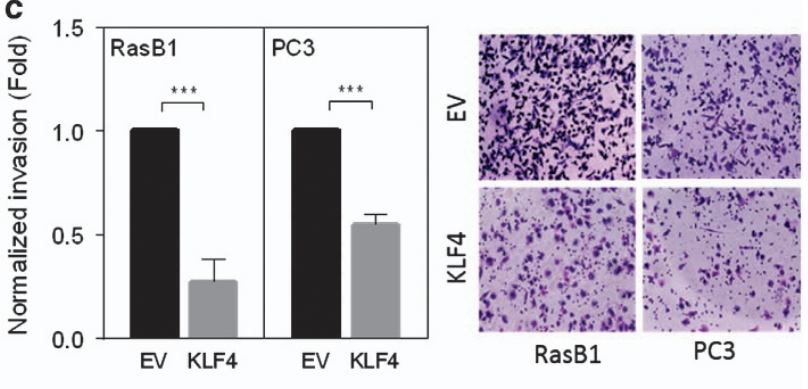

f

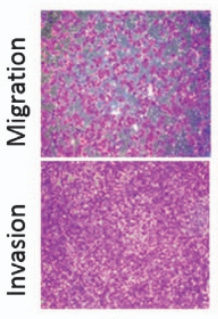

EV

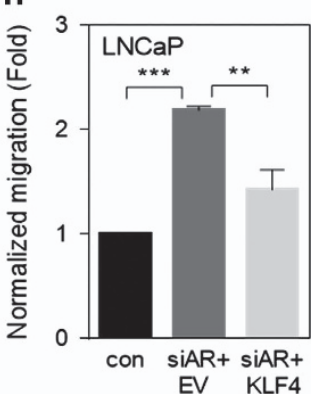

i

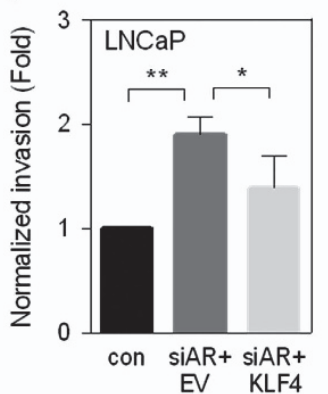

j

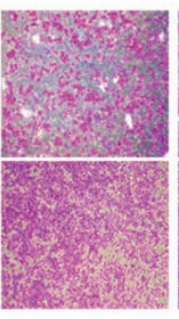

$A R+c o n$

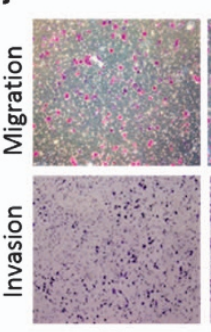

con

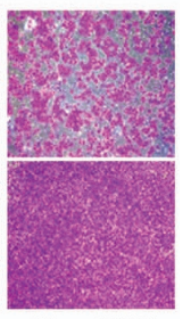

$A R+$ siKLF4

Figure 4. Androgen receptor (AR)-regulated Kruppel-like factor 4 (KLF4) reduced the malignant phenotypes of prostate cancer cells. (a) Proliferation of RasB1 cells stably overexpressing KLF4 or an empty vector (EV). All experiments were performed in triplicate and the data are presented as the mean \pm s.e.m., $n=6$. (b) Normalized colony-formation assay for RasB1 cells expressing KLF4 or the EV (left). Normalized colonies were counted with 10 microscopic images at $\times 100$ magnification after 14 days (right). All the experiments were performed in triplicate and the data are presented as the mean \pm s.e.m. Scale bar, $100 \mu \mathrm{m}$. (c) Normalized invasion of RasB1 and PC3 cells stably transfected with KLF4 or EV (left). The cells were plated in a serum-free medium above Matrigel transwell filters, with an attractant in the lower well. At $16 \mathrm{~h}$, the cells that had migrated through the filter to invade the lower well were quantified in five medium-power fields (right). All the experiments were performed in triplicate and the data are presented as the mean \pm s.e.m., $n=3$. (d and e) Normalized migration (d) and invasion (e) of RasB1 cells expressing AR or the EV following KLF4 or scramble control (con) SMARTpool siRNA expression. All the experiments were performed in triplicate and the data are presented as the mean \pm s.e.m., $n=3$. ${ }^{* * *} P<0.001,{ }^{* * * *} P<0.0001$. (f) Images of migration (d) and invasion (e). (g) Proliferation of LNCaP cells transfected with siAR or control siRNA following KLF4 or EV expression. All the experiments were performed in triplicate and the data are presented as the mean \pm s.e. of the mean, $n=6$. (h and $\mathbf{i})$ Normalized migration (h) and invasion (i) of LNCaP cells expressing siAR or control siRNA following KLF4 or EV expression. All the experiments were performed in triplicate and the data are presented as the mean \pm s.e.m., $n=3 .{ }^{*} P<0.05,{ }^{* *} P<0.01,{ }^{* * *} P<0.001$. (j) Images of migration (h) and invasion (i).

Figure 3. Kruppel-like factor 4 (KLF4) directly binds to the androgen receptor (AR) promoter and regulates its transcription activity. (a) Immunoblots for detecting AR and KLF4 protein expression in RasB1 and PC3 cells following KLF4 or control empty vector (EV) expression. (b) Immunoblots for detecting AR and KLF4 levels in 22Rv1 and LNCaP cells following KLF4 or luciferase (Luc) shRNA introduction. (c) Schematic of predicted KLF4-responsive elements (REs) and an introduced binding site mutant in promoter reporter constructs of the human $A R$ promoter at -347 bp upstream and $+312,+404,+647$ and +907 bp downstream. (d and e) Chromatin immunoprecipitation assay for KLF4 binding to predicted KLF4 REs in the AR promoter region measured in LNCaP-AR cells treated with $10 \mathrm{nM}$ DHT (d) and $10 \mu \mathrm{m}$ MDV3100 (e) for $4 \mathrm{~h}$. The binding activity of each protein to each site is given as a percentage of the total input normalized to each lgG. (f and $\mathbf{g}$ ) Activity of an RFP reporter gene containing KLF4 REs (K1 and K4) from the $A R$ promoter. Expression of the transiently transfected reporter gene, normalized to gene expression from a transfected control vector, was assayed in LNCaP-AR cells following $24 \mathrm{~h}$ treatment with $10 \mathrm{nM}$ DHT (f) or $10 \mu \mathrm{M}$ MDV3100 (g). Relative median fluorescence intensity (MFI) of the reporter protein following DHT or MDV3100 treatment. (h) The RasB1 cells were transiently cotransfected with wild-type (WT) or KLF4 RE-mutated K1- and K4-RFP reporters with KLF4 expression vectors for $48 \mathrm{~h}$. The MFI was measured through fluorescence-activated cell sorting and normalized to the value of the EV. All the experiments were performed in triplicate and the data are presented as the mean \pm s.e.m., $n=3$. NS, nonsignificant, ${ }^{* *} P<0.01,{ }^{* * *} P<0.001$. 
a

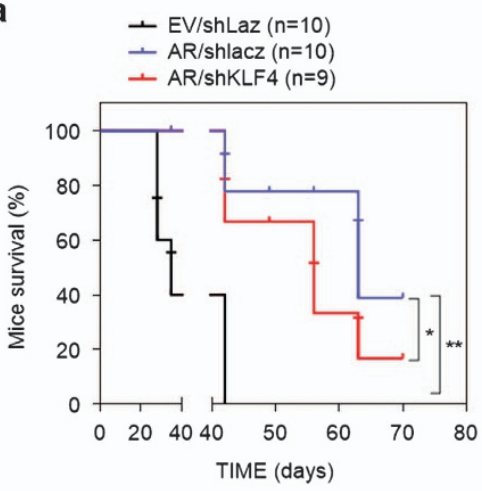

c

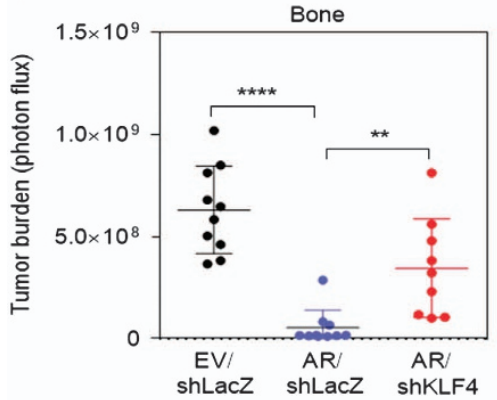

b

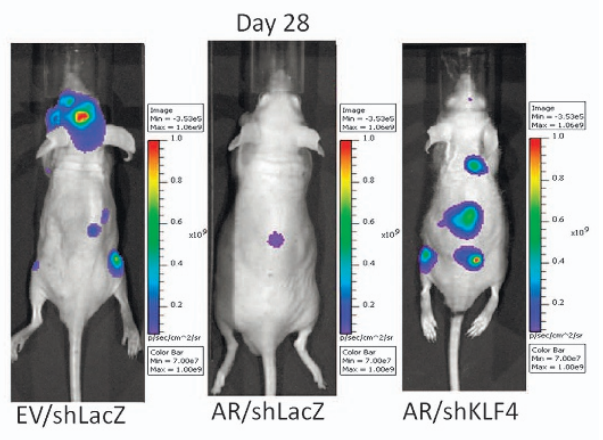

d

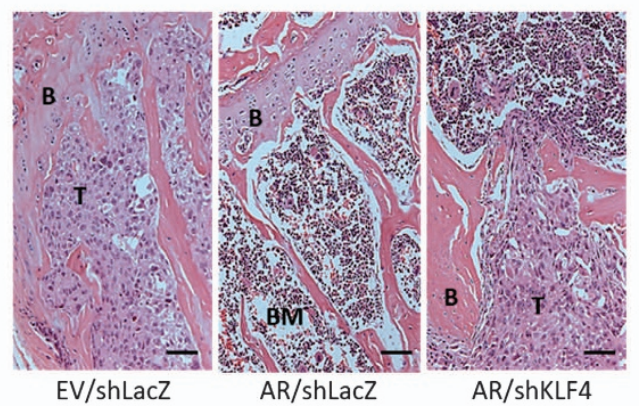

Figure 5. Reduced Kruppel-like factor 4 (KLF4) expression increased bone metastasis in association with androgen receptor (AR) expression. (a) Survival rates of tumor-bearing mice with RasB1/empty vector (EV) or RasB1/AR cells expressing a control shLacZ or KLF4 shRNA vector (RasB1/EV/shLacZ, $n=10$; RasB1/AR/shLacZ, $n=10$; RasB1/AR/shKLF4, $n=9$ ). (b) Representative bioluminescence imaging (BLI) in tumorbearing mice at day 28 after injection with the same cells as in a. (c) Quantification of the BLI signals of the mice with bone metastases in $\mathbf{b}$. (d) Representative histological images of bone metastases in tumor-bearing mice inoculated with the same cells as in b. B, bone; BM, bone marrow; T, tumor. Scale bar, $100 \mu \mathrm{m}$. Log-rank test was used for survival curve analysis. ${ }^{*} P<0.05,{ }^{* *} P<0.01,{ }^{* * * *} P<0.0001$.

overexpressing both AR and FOXA1 (Figure 2e). To characterize the specificity of AR binding to the KLF4 promoter region, we introduced point mutations in the ARE regions (Figure 2a); we observed that mutations in ARE2 disrupted the ability of AR and FOXA1 to stimulate KLF4 promoter activity in reporter assays (Figure 2f). These data are consistent with the mechanism that AR activates KLF4 transcription through direct physical interaction with the KLF4 promoter.

KLF4 induces AR expression by reciprocally interacting with the $A R$ promoter

To determine whether KLF4 can regulate AR expression, thereby creating a regulatory loop between AR and KLF4, we analyzed the consequences of modulating KLF4 expression on AR expression in prostate cancer cells. AR mRNA levels increased in RasB1 and PC3 cells transfected with an ectopic KLF4 expression vector (Supplementary Figure S1A). Consistently, the AR and KLF4 protein levels also increased in RasB1 and PC3 cells transfected with the ectopic KLF4 expression vector (Figure 3a). Notably, the AR and KLF4 mRNA levels decreased in 22Rv1 and LNCaP cells transfected with small interfering RNA (siRNA) against KLF4 (siKLF4; Supplementary Figure S1B). Moreover, the AR and KLF4 protein levels decreased in cells transfected with a KLF4 shRNA knockdown vector (shKLF4; Figure 3b). These data strongly support the presence of positive feedback between $A R$ and KLF4 in prostate cancer cells.

To further investigate this mechanism and determine whether KLF4 regulates $A R$ expression at the transcriptional level, we analyzed the $A R$ promoter for the presence of KLF4 homologous binding sites and identified five putative sites (Figure $3 \mathrm{C}$ ). To assess the ability of KLF4 to bind to the $A R$ promoter, we performed ChIP assays. KLF4/AR chromatin complexes were immunoprecipitated using an anti-KLF4 antibody from nuclear extracts of LNCaP-AR cells after DHT treatment. By using qRT-PCR, we then analyzed the KLF4-responsive element (RE) region of $A R$. We noted significantly increased nuclear KLF4-binding signals at putative $\mathrm{K} 1$ and $\mathrm{K} 4$ sites in DHT-treated cells compared with those in untreated cells (Figure 3d). By contrast, decreased nuclear KLF4-binding signals were observed at the same sites after MDV3100 treatment (Figure 3e). To determine whether the KLF4-binding sites of the $A R$ promoter (K1 and K4) are functional, we conducted reporter assays by using a construct with a KLF4-RE at the K1 and K4 sites incorporated into an RFP reporter. The reporter activity increased in response to DHT (Figure $3 f$ ) and decreased after MDV3100 treatment (Figure 3g). The introduction of mutations in the KLF4 binding sites in the $A R$ promoter abolished the effects of KLF4 on $A R$ transcription (Figure $3 \mathrm{~h}$ and Supplementary Figure S1C). We additionally treated AR-positive LNCaP and 22Rv1 cells with siKLF4 and observed that the $A R$ promoter reporter activity decreased in the presence of siKLF4 (Supplementary Figure S1D). These data demonstrate that KLF4 directly binds to the $A R$ promoter and regulates $A R$ expression.

KLF4 inhibits cell growth and motility by upregulating AR expression

To assess the role of KLF4 in human prostate cancer progression, we stably expressed KLF4 in RasB1 and PC3 cells (as confirmed by immunoblots in Figure 3a). KLF4 overexpression significantly reduced the growth rate of these cells in vitro (Figure $4 a$ and Supplementary Figure S2A). Notably, when we expressed KLF4 in RasB1 cells, the colony formation of these cells decreased in three-dimensional growth assays in soft agar (Figure 4b). Moreover, when we treated AR-positive LNCaP and 22Rv1 cells with shKLF4 (knockdown effect as confirmed by immunoblots in 
Figure 3b), the colony formation of these cells increased (Supplementary Figure S2B). We further examined the functional relevance of KLF4-mediated reduction in the migration and invasion of RasB1 and PC3 cells. The cells expressing KLF4 had reduced cell motility compared with cells carrying the control vector, according to migration (Supplementary Figure S2C) and invasion (Figure 4c) assays. Moreover, the motility of the
AR-positive LNCaP-AR cells through transwells was significantly induced when siKLF4 was expressed (Supplementary Figure S2D). To further confirm that cell motility was reduced by the AR-dependent induction of KLF4 expression, RasB1 and PC3 cells were stably transfected with $A R$ and subsequently transfected with siKLF4. The cells overexpressing AR had decreased invasiveness and migration compared with cells expressing the empty a

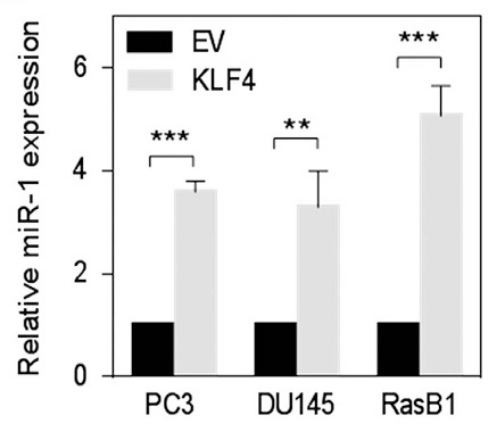

C
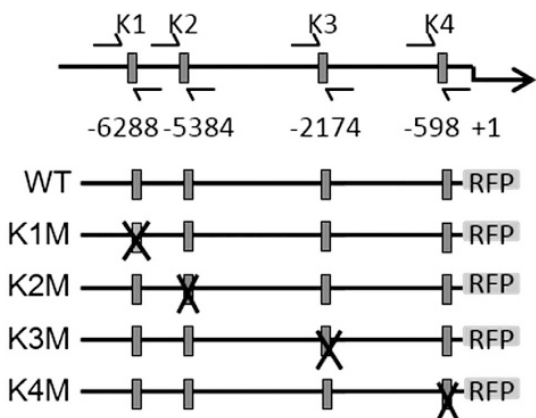

d

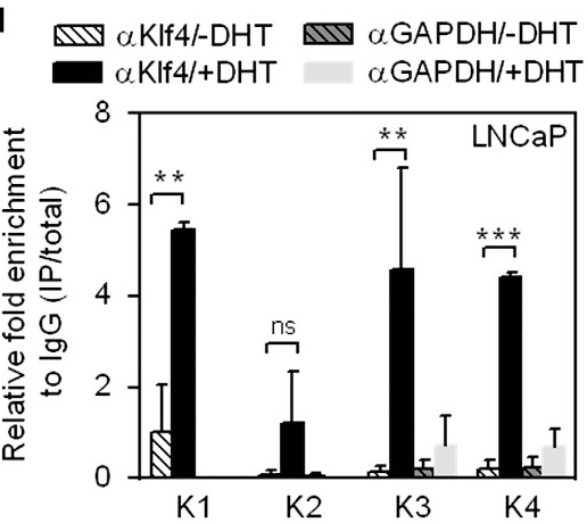

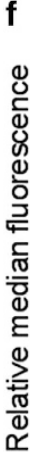

pri-miR-1-2 promter-RFP

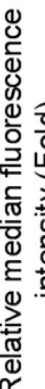

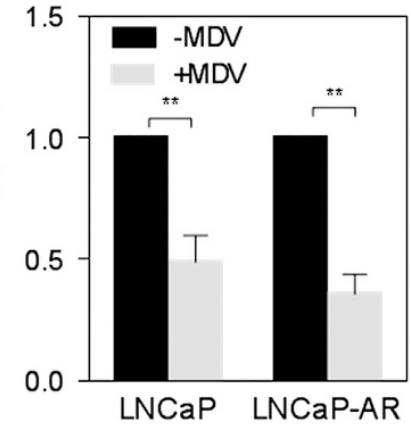

b
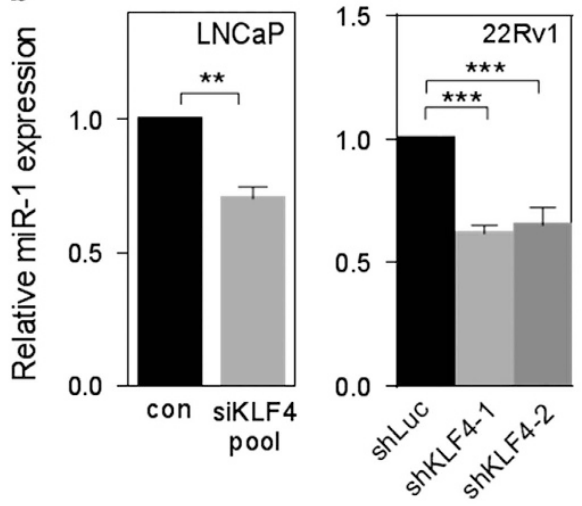

Has-pri-miR-1-2

promoter (GRCh37:18)

Wild type Mutant

$\mathrm{K} 1 \mathrm{M}$ CACCCC $\rightarrow$ GGTACC

K2M AGGGTG $\rightarrow$ GGTACC

$\mathrm{K} 3 \mathrm{M}$ AGGGTG $\rightarrow$ GGTACC

$\mathrm{K} 4 \mathrm{M}$ CACCCA $\rightarrow$ GGTACC

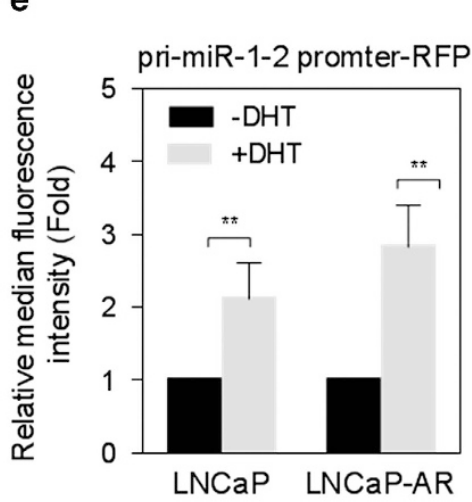


vector, whereas cell invasion and migration were induced when the AR-expressing cells were treated with siKLF4 (Figures $4 d-f$ and Supplementary Figures S2E-G). We next performed AR knockdown combined with KLF4 overexpression or AR overexpression combined with KLF4 knockdown and analyzed the effects on proliferation and motility of LNCaP and 22Rv1 cells. Remarkably, AR knockdown in AR-positive LNCaP cells induced cell proliferation, migration and invasion, whereas KLF4 overexpression in AR-knockdown cells decreased cell proliferation, migration and invasion (Figures $4 \mathrm{~g}-\mathrm{j}$ ). Moreover, significant cell proliferation and motility were induced in AR-positive 22Rv1 cells when siKLF4 was expressed, whereas AR overexpression in KLF4-knockdown cells decreased cell proliferation, migration and invasion (Supplementary Figures $\mathrm{S} 2 \mathrm{H}-\mathrm{K}$ ). These results demonstrate that KLF4 and AR can compensate each other in the negative regulation of proliferation, migration and invasion.

These results were further supported by in vivo experiments. We intracardially administered AR-overexpressing RasB1 cells to mice and noted an increase in survival rates (Figure $5 \mathrm{a}$ ) and a considerable decrease in bone metastasis (Figure 5b). However, the administration of AR-expressing cells with shKLF4 significantly increased bone metastasis and decreased survival rates compared with mice injected with control shRNA-harboring cells (Figures $5 a-d$ ), confirming that the AR-dependent induction of KLF4 expression is associated with bone metastasis. In summary, the proliferation and metastatic abilities of prostate cancer cells are closely regulated by KLF4 expression, supporting its role as a tumor suppressor in prostate cancer.

KLF4 induces miR-1 expression by directly binding to primary miR-1 promoter

To investigate the tumor-suppressor role of KLF4, we focused on identifying KLF4 target substrates potentially involved in tumorigenesis prevention. We previously showed that activated AR can directly target miR-1 and that miR-1 functions as an inhibitor of prostate cancer bone metastasis. ${ }^{20}$ Accordingly, we analyzed the relationships between the genes associated with high miR-1 expression and androgen-responsive gene signatures $^{27}$ in the Taylor Prostate Cancer Dataset. ${ }^{28}$ By using a bioinformatics approach, GSEA, we determined an association between KLF4 expression and AR-induced miR-1 expression (Supplementary Figures S3A,B). Next, we determined whether miR-1 levels are associated with KLF4 expression in prostate cancer. First, we observed that KLF4 overexpression in AR-negative prostate cancer cell lines increased miR-1 expression (Figure 6a). KLF4 inhibition through the transient transfection with siKLF4 in LNCaP or stable transfection with shKLF4 in 22Rv1 prostate cancer cells reduced endogenous miR-1 levels (Figure 6b). Thus, we hypothesized that KLF4 stimulates miR-1 expression by interacting with the primary miR-1 stem-loop promoter. We then analyzed the primary miR-1 stem-loop promoter for a homologous RE of KLF4 and observed four candidate binding elements for KLF4 in the upstream promoter region of the primary miR-1-2 gene (that is,
pri-miR-1-2; Figure 6c). To identify which of these sites is required to stimulate miR-1 expression, we performed ChIP assays in LNCaP cells left untreated or treated with DHT by using an anti-KLF4 or control anti-GAPDH antibody. qRT-PCR analyses indicated a significant increase in KLF4-binding signals at the K1, K3 and K4 sites after DHT treatment (Figure 6d). We then performed reporter assays in prostate cancer cell lines by using the wild-type pri-miR-1-2 stem-loop promoter, containing KLF4-REs driving RFP reporter expression. Compared with untreated cells, reporter gene activity significantly increased in DHT-treated LNCaP and LNCaPAR cells (Figure 6e), whereas it decreased in MDV3100-treated cells (Figure 6f). Moreover, KLF4 overexpression increased wildtype pri-miR-1-2 reporter activity; however, a single mutation in each of the four KLF4 binding sites could disrupt the ability of KLF4 to induce pri-miR-1-2 reporter activity (Figure 6g). These results indicate that KLF4 regulates miR-1 expression through the direct binding of KLF4 to the pri-miR-1-2 promoter region.

In a previous report, we demonstrated that AR can promote pri-miR-1-2 transcription. ${ }^{20}$ Here, to study the contribution of KLF4 and $A R$ in pri-miR-1-2 transcription, we first determined miR-1 levels in AR-positive cells after knocking down AR, KLF4 or both. Our qRT-PCR results indicated that the simultaneous knockdown of siAR and siKLF4 resulted in the highest downregulation of miR-1 expression (Supplementary Figure S3C, left). We also examined miR-1 levels in AR-negative cells after overexpressing AR and/or KLF4. Cotransfection with AR and KLF4 induced the highest miR-1 levels (Supplementary Figure S3C, right). Moreover, the cotransfection of AR-positive LNCaP cells with the pri-miR-1-2 promoter reporter and siAR, siKLF4 or both siRNA induced the lowest reporter activity (Supplementary Figure S3D, left), whereas overexpression of both the KLF4 and AR expression vectors in AR-negative RasB1 cells induced the highest reporter activity (Supplementary Figure S3D, right). These results suggest that AR and KLF4 cooperatively promote the transcription of pri-miR-1-2.

KLF4 expression is positively correlated with AR and miR-1 levels in clinical prostate cancer samples

To further study the link between KLF4 and its targets AR and miR-1 in clinical samples, we analyzed 22 independent prostate tumor tissue samples collected from the Wan Fang Hospital, Taipei Medical University (Taiwan). The samples were divided into two groups of low and high levels of both AR and miR-1 expression, as measured using qRT-PCR. Analysis of variance confirmed that KLF4 expression levels were significantly higher in the groups with high AR or miR-1 expression levels (Figure 7a). Similarly, KLF4 expression was positively correlated with AR and miR-1 expression according to a Pearson coefficient correction analysis (Figure 7b). Through immunohistochemistry, we observed that tumor tissues with high miR-1 expression levels exhibited strong cytoplasmic and nuclear staining for KLF4 (Figure 7c-top, left). Moreover, strong nuclear staining for AR was observed in the same tissue samples (Figure $7 c$-top, right). Notably, the expression of both

Figure 6. Kruppel-like factor 4 (KLF4) transcriptionally upregulates microRNA (miR)-1 by binding to the pri-miR-1-2 stem-loop promoter. (a) Quantitative reverse-transcription PCR (qRT-PCR) analysis of miR-1 in LNCaP, LNCaP-AR and RasB1 cells expressing KLF4 or an empty vector (EV) for $48 \mathrm{~h}$. (b) qRT-PCR analysis of miR-1 in LNCaP or 22Rv1 cells following the introduction of a control or KLF4 SMARTpool siRNA (left) or a control luciferase (Luc) or KLF4 shRNA vector (right) for $48 \mathrm{~h}$. (c) Schematic of the predicted KLF4-binding sites (K1 to K4) and an introduced binding site mutant in the human pri-miR-1-2 stem-loop promoter at $-6288,-5384,-2174$ and -598 bp upstream. (d) Chromatin immunoprecipitation assays for KLF4 binding to predicted KLF4 binding sites measured in LNCaP cells treated with 10 nM DHT for $4 \mathrm{~h}$. The binding activity of each protein to each site is given as a percentage of the total input normalized to each IgG. (e and f) LNCaP and LNCaP-AR cells were transiently transfected with a pri-miR-1-2 stem-loop promoter reporter following $48 \mathrm{~h}$ treatment with $10 \mathrm{~nm} \mathrm{DHT} \mathrm{(e)} \mathrm{or} 10 \mu \mathrm{M}$ MDV3100 (f). The relative median fluorescence intensity (MFI) of the reporter protein following DHT or MDV3100 treatment is shown. (g) The LNCaP-AR cells were transiently cotransfected with wild-type (WT) or mutated KLF4 binding site (K1M to K4M) reporters with KLF4 or an EV for $48 \mathrm{~h}$. The MFI was measured through fluorescence-activated cell sorting and normalized to the value of the EV. All the experiments were performed in triplicate and the data are presented as the mean \pm s.e.m., $n=3$. NS, nonsignificant, ${ }^{* *} P<0.01,{ }^{* * *} P<0.001$. 
KLF4 and AR was low in tumor tissue samples with low miR-1 expression (Figure 7c-bottom).

We next validated the relationship between AR, KLF4 and miR-1 expression in human prostate cancer tissues from the Taylor Prostate Cancer Dataset and The Cancer Genome Atlas. The analysis of variance results indicated that KLF4 expression was induced in tissue samples with high miR-1 and AR levels (Figures 7d and e). We also noted that KLF4 and miR-1 levels were negatively associated with cancer grading (Supplementary

a

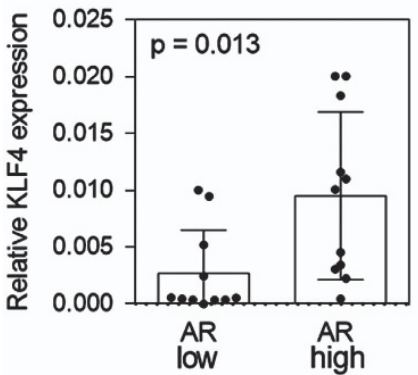

b

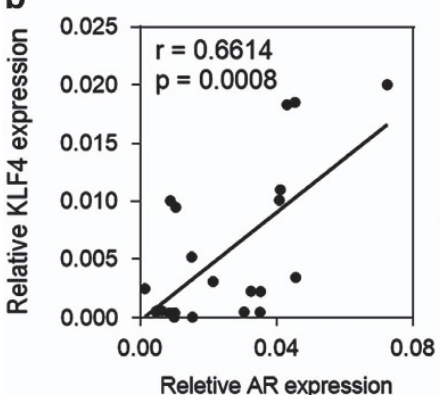

Reletive AR expression

C

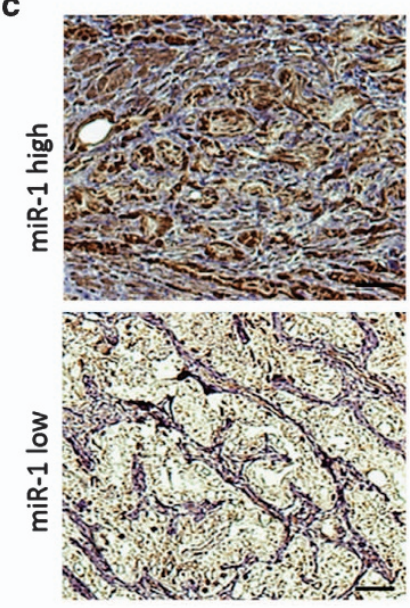

KLF4

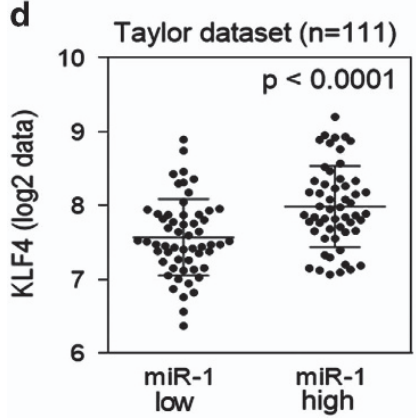

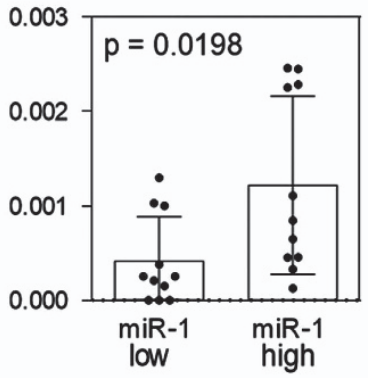
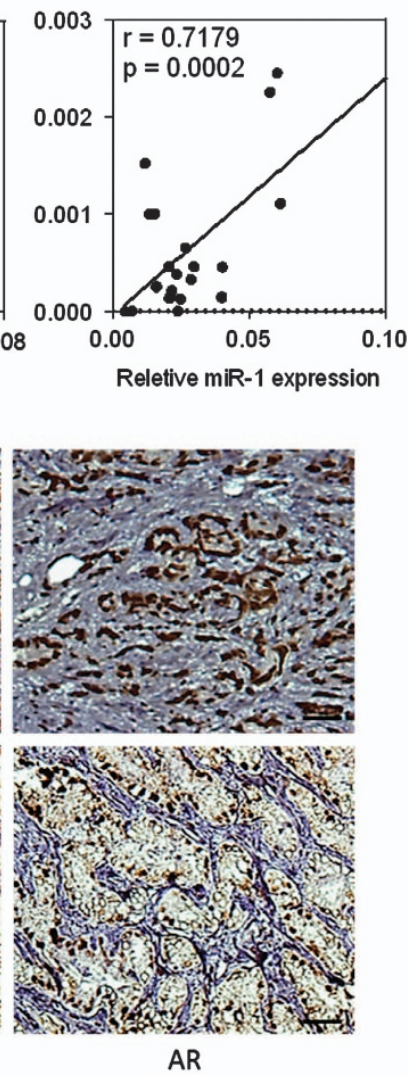

AR

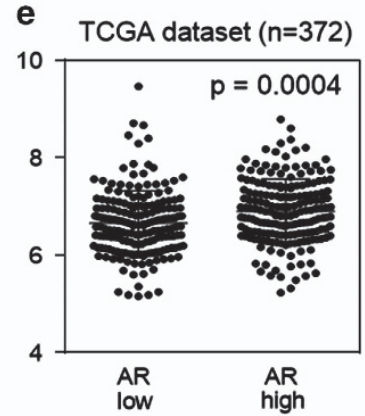

Figures $\mathrm{S} 4 \mathrm{~A}, \mathrm{~B})$. To confirm the positive correlation between KLF4 and $A R$ signaling in prostate cancer, clinical samples from the Taylor Prostate Cancer Dataset were divided into two groups with either high or low KLF4 expression, based on a measure of the relative mRNA expression using z-scores. A z-score analysis of the mean showed higher KLF4 expression in samples with upregulated androgen-dependent genes ${ }^{27}$ (Supplementary Figure S4C). Furthermore, GSEA using two androgen-responsive gene sets ${ }^{24,27}$ showed a positive association between high KLF4 expression and androgen-responsive gene sets in clinical samples from the Taylor Prostate Cancer Dataset (Supplementary Figures S4D,E). Therefore, prostate cancer samples with increased AR levels had increased KLF4 expression. Taken together, our findings identify a regulatory mechanism by which AR upregulates KLF4 expression directly and transcriptionally; subsequently, KLF4 increases miR-1 expression levels and sustains suppressed prostate tumorigenesis. In addition, $A R$ expression is reciprocally upregulated by KLF4, creating an amplifying retropositive loop.

\section{DISCUSSION}

KLF family proteins are transcription factors involved in the regulation of several cellular processes, including proliferation, apoptosis, differentiation, inflammation, migration and tumor formation. ${ }^{1}$ Our studies have focused on the role of KLF4 in prostate carcinogenesis, particularly in metastasis inhibition. AR is a member of the family of intracellular steroid hormone receptors and functions as a ligand-dependent transcription factor. ${ }^{29}$ Here, we identified a self-reinforcing regulatory loop for the KLF4-AR axis that involves the AR-induced transcriptional stimulation of the KLF4 promoter, indicating that KLF4 is a candidate target gene for $\mathrm{AR}$ and $A R$ is a candidate target gene for KLF4. In concordance with the tumor-suppressive effect mediated by KLF4 activation, we demonstrated that AR-induced KLF4 expression negatively regulates the proliferation and metastatic abilities of prostate cancer cells. We further showed that KLF4 increases miR-1 expression levels and sustains as a tumor suppressor of prostate cancer. KLF4 inhibition in several cancer types, including prostate cancer, may contribute to cellular hyperproliferation and malignant transformation. 4, $4,21,30,31$ Our previous report showed that KLF4 is a direct transcriptional inhibitor of SLUG expression in prostate cancer cells, ${ }^{2}$ suggesting a role of KLF4 in inhibiting cell migration and invasion in prostate cancer. Regarding the cell cycle, KLF4 can cause either G1 or G2 arrest in different cell types. $^{32-34}$ Studies have indicated that KLF4 functions as a tumor suppressor in regulating cell proliferation and apoptosis on the basis of the modulation of downstream genes, such as those of $\mathrm{p} 27^{30} \mathrm{p} 21,{ }^{35} \mathrm{p} 57,{ }^{36}$ cyclin D1 (ref. 37 ) and cyclin B1, ${ }^{38}$ and that

Figure 7. Increased Kruppel-like factor 4 (KLF4) expression is positively correlated with androgen receptor (AR) and microRNA (miR)-1 expression in prostate cancer patients. (a) KLF4 levels in two tissue sample groups divided on the basis of AR and miR-1 levels ( $n=11$ per group). (b) Pearson correlation coefficient analysis of the association between the mean KLF4 and mean AR and miR-1 mRNA expression levels in independent primary prostate samples $(n=22)$. Significance was determined according to the Gaussian population (Pearson) and a two-tailed test. (c) Immunohistochemistry with KLF4- and AR-specific antibodies in prostate cancer tissue sections with different miR-1 levels. Scale bars represent $100 \mu \mathrm{m}$. (d) KLF4 levels in two tissue sample groups containing 98 primary tumor and 13 distant metastasis samples separated on the basis of miR-1 expression from the Taylor Prostate Cancer Dataset. Significance was determined using the Student's $t$-test. (e) KLF4 levels in two tissue sample groups containing 372 primary tumor samples separated on the basis of AR expression from the The Cancer Genome Atlas (TCGA) data set. Significance was determined using the Student's $t$-test. 
KLF4 has potent tumor suppressor-like function. miR-1 has been described as a suppressor of prostate cancer. ${ }^{16,17,19}$ We previously reported the loss of AR-mediated activation of miR-1 and subsequent activation of SRC-stimulated prostate cancer bone metastasis. ${ }^{20}$ Although activated AR stimulates miR-1 transcription by binding to its promoter, ${ }^{20}$ our present results support a model where AR might function as a transcription factor that activates the KLF4-miR-1 signaling pathway to persist the tumorsuppressive role of miR-1. This finding is consistent with our results showing reduced KLF4 and miR-1 levels were negatively associated with elevated cancer grading.

A combination of androgen-deprivation therapy and surgical prostatectomy or radioablation has been used to treat therapynaive patients with prostate cancer. ${ }^{39,40}$ Although the initial response is typically efficient, almost all patients develop castration-resistant prostate cancer, frequently leading to patient death. ${ }^{41}$ Our current study provides a novel explanation of this phenomenon: androgen-deprivation therapy results in AR signaling inhibition, leading to KLF4 and miR-1 repression and potentially contributing to castration-resistant prostate cancer. Alternatively, AR inhibition may cause the inactivation of KLF4-repressing tumorigenesis pathway components that affect the biological functions of cells. Several lines of evidence have suggested that during prostate cancer development, AR has a dual function as an oncogene ${ }^{42}$ and a tumor suppressor. ${ }^{43}$ Moreover, the transcription profiles of AR could be very different between androgen-dependent and -independent prostate cancers. ${ }^{24}$ Our current study supported the tumor suppressor role of AR by indicating an AR-KLF4 positive feedback loop, which can inhibit malignant phenotypes in AR-negative or androgeninsensitive prostate cancer cells (PC3 and RasB1). We also studied the underlying mechanisms contributing to the resistance to androgen-deprivation therapy and poor AR antagonist efficacy in advanced prostate cancer. Our results may aid in understanding individual intervariability in drug responses to consequently improve drug therapies: by modulating KLF4 expression to repress cancer malignancy, an alternative target may be identified to improve the efficacy of current prostate cancer therapy modalities.

In conclusion, KLF4 expression is directly and transcriptionally upregulated by $A R$, and $A R$ expression is reciprocally upregulated by KLF4. This study highlights the role of the KLF4-AR axis in androgen deprivation and reveals pivotal mechanisms responsible for castration-resistant prostate cancer development. Despite the gaps in the understanding of KLF4 and its interplay with cell motility and proliferation pathways, we present a novel function for the KLF4-miR-1 axis-a new putative predictive and surveillance biomarker of antiandrogen therapy for advanced prostate cancer.

\section{MATERIALS AND METHODS}

\section{Cells, reagents and constructs}

Androgen-dependent LNCaP-AR (parental LNCaP overexpressing wild-type AR) and metastatic RasB1 (DU145 expressing a constitutively active Ras) cell lines were obtained from $\mathrm{Dr}$ Kathleen Kelly $(\mathrm{NCl} / \mathrm{NIH}$, Bethesda, MD, USA) and maintained as described previously. 20,44-48 DU145, PC3, LNCaP and 22Rv1 cell lines were from the American Type Culture collection (Manassas, VA, USA). All the cells were cultured in the RPMI 1640 medium supplemented with $10 \%$ FBS (fetal bovine serum). After DHT treatment (10 nм; Sigma, St Louis, MO, USA), cells were cultured for $24 \mathrm{~h}$ in a $10 \%$ charcoal-stripped FBS-containing medium. Some cells were treated with the AR antagonist MDV3100 (Selleck, Houston, TX, USA) at $10 \mu \mathrm{m}$ for $24 \mathrm{~h}$ in a $10 \%$ FBS-containing medium. The cells with stable or transient AR, FOXA1, or KLF4 expression were established through transfection with AR, FOXA1 or KLF4 expression vectors, respectively; an empty vector, $\mathrm{pCDH}-$ CMV-MCS-EF1-Puro (System Biosciences, Palo Alto, CA, USA) with a puromycin-selectable marker, was used as a control. The siRNA (scramble and siKLF4) and shRNA vectors (shLuc and shKLF4) were purchased from Thermo Scientific (Dharmacon SMARTpool siRNA Reagents; Waltham, MA,
USA) and the RNAi Core Lab (Academia Sinica, Taipei, Taiwan). Transient transfection of the plasmids and siRNAs was performed using the X-tremeGENE HP DNA transfection reagent (Roche, Clovis, CA, USA) and Lipofectamine RNAiMAX (Invitrogen, Waltham, MA, USA), respectively. By using GRCh37, KLF4 binding sites were located on human pri-miR-1-2 and $A R$ on chromosomes 18 and $\mathrm{X}$, respectively, and $A R$ binding sites were located on human KLF4 on chromosome 9 (Supplementary Table S1). The $A R, K L F 4$ and pri-miR-1-2 promoters with AR- and KLF4-binding site-RFP reporter vectors were constructed using the Clone-it Enzyme Free Lentivectors Kit (System Biosciences). All the primers used for these constructs are listed in Supplementary Table S2. All the constructs were verified using DNA sequence analysis.

\section{Western blot analysis}

The cells grown on six-well plates $\left(10^{6}\right.$ cells/well) were lysed in $150 \mu$ l of RIPA buffer containing complete protease inhibitors (Roche) and phosphatase inhibitors (Roche), $25 \mathrm{~mm} \beta$-glycerophosphate, $10 \mathrm{~mm}$ sodium fluoride and $1 \mathrm{~mm}$ sodium vanadate. Twenty micrograms of protein was separated per lane through SDS-gel electrophoresis. After transfer to polyvinylidene fluoride membrane, the blots were blocked with $5 \%$ bovine serum albumin in phosphate-buffered saline plus Tween 20. Primary antibodies were incubated overnight at $4{ }^{\circ} \mathrm{C}$, and secondary antibodies were incubated at room temperature for $1 \mathrm{~h}$ as indicated in Supplementary Table S3.

\section{qRT-PCR analysis}

We measured KLF4, AR and miR-1 expression in the human prostate cancer cell lines, with or without DHT or MDV3100 treatment and AR or KLF4 overexpression, by using qRT-PCR. Total RNA was isolated using the mirVana PARIS RNA isolation system (Ambion, Waltham, MA, USA). For reverse transcription, $3 \mu \mathrm{g}$ of total RNA was used with the SuperScript III kit (Invitrogen). In the amplification step, SYBR green PCR master mix (Applied Biosystems, Waltham, MA, USA) was used. For all primer pairs, the thermocycler was run for an initial $95^{\circ} \mathrm{C}$ incubation for $10 \mathrm{~min}$, followed by 40 cycles with $95^{\circ} \mathrm{C}$ for $15 \mathrm{~s}$ and $60^{\circ} \mathrm{C}$ for $1 \mathrm{~min}$. All the reactions were normalized to human GAPDH and run in triplicate. All the primers used for PCR are listed in Supplementary Table S4. miR-1 RT-PCRs were performed using the TaqMan MicroRNA Assays kit (Applied Biosystems). All the values were normalized to a human SNORD48 endogenous control and run in triplicate.

\section{ChIP assay}

The cells were treated with or without DHT (10 nm) for $4 \mathrm{~h}$. The cultured cells $\left(10^{7}\right)$ were cross-linked with $1 \%$ formaldehyde at room temperature for $15 \mathrm{~min}$. Fixation was quenched with glycine, and the cells were washed twice with cold phosphate-buffered saline containing a complete protease inhibitor (Roche). The cell pellets were resuspended in cell lysis buffer and incubated on ice for $15 \mathrm{~min}$. Nuclei were collected by centrifugation at $10^{4}$ r.p.m. and $4{ }^{\circ} \mathrm{C}$ for $10 \mathrm{~min}$ and resuspended in nuclear lysis buffer. Chromatin was sheared using a sonicator (Branson Sonifier 250, Dietzenbach, Germany) with a microtip in a $20 \mathrm{~s}$ burst followed by $1 \mathrm{~min}$ of cooling on ice for a total sonication time of $5 \mathrm{~min}$ per sample. This procedure results in DNA fragment sizes of approximately 100 to $300 \mathrm{bp}$. Sheared chromatin was divided to perform immunoprecipitation with a rabbit IgG antibody (Santa Cruz Biotechnology, Dallas, TX, USA) or primary antibody at $4{ }^{\circ} \mathrm{C}$ overnight. Immunoprecipitation, washing, elution, reverse cross-linking and DNA purification steps were performed according to Millipore's protocol. A qRT-PCR was performed in triplicate with $2 \mu \mathrm{l}$ of eluted chromatin. ChIP antibodies and PCR primers are listed in Supplementary Table S5. Predictions for transcription factor-binding sites within promoter regions were adopted from the AliBaba 2.1 program (gene-regulation.com).

\section{Promoter reporter assay}

Promoter function was analyzed through FACS (fluorescence-activated cell sorting), and relative median fluorescent intensity was measured from the first peak of fluorescence, as described previously. ${ }^{20,47,48}$ The cells were treated with or without $10 \mathrm{~nm}$ DHT and $10 \mu \mathrm{m}$ MDV3100 for $48 \mathrm{~h}$. The median fluorescent intensity for RFP was measured through FACS by using FACSDiva software and normalized to the value of the vehicle. Three independent experiments were performed in triplicate. 
Proliferation assay

The PC3 and RasB1 cells were transfected with the KLF4 expression vector at a density of 2000 cells/well. Each day, the cells were stained with $0.5 \%$ crystal violet fixative solution for $15 \mathrm{~min}$, washed with distilled water and allowed to air dry. At the end of the experiment, crystal violet was dissolved by adding $100 \mu \mathrm{l}$ of $50 \%$ ethanol containing $0.1 \mathrm{~m}$ sodium citrate to each well, and absorbance was measured at $550 \mathrm{~nm}$ on an ELISA reader (Biocompare, South San Francisco, CA, USA).

\section{Colony-formation assay}

Colony-formation assays were performed using a starting cell count of $5 \times 10^{4}$ cells/well. Single-cell suspensions of KLF4-transfected RasB1 cells or shKLF4 vector-transfected LNCaP-AR or DU145 cells were seeded in six-well plates in the RPMI 1640 medium supplemented with 10\% FBS. The colonies were counted on day 21 after plating in triplicate and normalized with a control shLuc vector.

\section{Invasion and migration assays}

For invasion and migration assays, KLF4 or AR expression vectortransfected PC3 and RasB1 cells with a control siRNA or siKLF4 or siKLF4-transfected LNCaP-AR cells were resuspended at a concentration of $2.5 \times 10^{5} \mathrm{cell} \mathrm{s} / \mathrm{ml}$ in a serum-free medium. We purchased Matrigel from BD Biosciences (San Jose, CA, USA) for the invasion assay. Matrigel-coated transwell dishes were prepared by adding $200 \mu \mathrm{l}$ of Matrigel diluted 10fold with the serum-free medium. The cells were plated at $2.5 \times 10^{5}$ per well in the serum-free medium above the Matrigel. The lower chamber was filled with $600 \mu \mathrm{l}$ of the serum-containing medium. The cells that had invaded the Matrigel-coated transwells after $12 \mathrm{~h}$ were fixed and stained with a $0.5 \%$ crystal violet fixative solution for $15 \mathrm{~min}$. The invading cells on the underside of the membrane were counted and quantified in five medium-power fields for each replicate in triplicate. In the migration assay, we used transwells without Matrigel, and the cells were fixed and stained as described for the invasion assay.

\section{Metastasis and survival assays in mice}

Animal experiments were performed according to the protocol approved by the Taipei Medical University Animal Care and Use Committee (Taiwan). To analyze metastasis, 7-week-old male nude mice (NLAC, Taipei, Taiwan) were intracardially administered either $10^{5}$ RasB1 human metastasis prostate cancer cells harboring a luciferase expression vector with a control empty or AR expression vector or $10^{5}$ AR-transfected RasB1 cells with a control shLacZ or shKLF4 expression vector. Bioluminescence imaging was performed double blinding at 28 days after injection as described previously. ${ }^{46}$ In all cases, the same mice were followed for up to 10 weeks after injection to measure the accumulation of bone metastases through bioluminescence imaging as well as the survival percentage. For survival studies, mice were killed upon $10 \%$ body weight loss, paralysis or head tilting.

\section{Immunohistochemistry}

We collected 22 independent primary prostate tumor samples from the Wan Fang Hospital, Taipei Medical University (Taiwan). The study was approved by the Wan Fang Hospital, Taipei Medical University Institutional Review Board (approval no.: N201512033) and performed according to the approved guidelines. Immunohistochemistry was performed using antiKLF4 (Sigma) and anti-AR (Epitomics, Burlingame, CA, USA) antibodies at 1:500 and 1:250 dilutions, respectively, as described in Supplementary Materials and Methods.

\section{Statistical analysis}

All the data are presented as the mean \pm s.e.m. Statistical calculations were performed on GraphPad Prism analysis tools (GraphPad Software, La Jolla, CA, USA). Differences between individual groups were determined using the Student's $t$-test or one-way analysis of variance, followed by Bonferroni's post test for comparisons among three or more groups. The method for determining the cutoff points was predecided by considering half the number of patients. A $P$-value of $<0.05$ was considered statistically significant.

\section{CONFLICT OF INTEREST}

The authors declare no conflict of interest.

\section{ACKNOWLEDGEMENTS}

This work was jointly supported by grants from the Ministry of Science and Technology of Taiwan (MOST 104-2320-B-038-038 and MOST 105-2628-B-038-006MY3 to MKS; MOST 104-2314-B-038-045-MY3 to Y-NL), the National Health Research Institutes of Taiwan (NHRI-EX105-10308BC to Y-NL) and Taipei Medical University-Wan Fang Hospital (105TMU-WFH-01 to MKS).

\section{REFERENCES}

1 McConnell BB, Yang VW. Mammalian Kruppel-like factors in health and diseases. Physiol Rev 2010; 90: 1337-1381.

2 Liu YN, Abou-Kheir W, Yin JJ, Fang L, Hynes P, Casey O et al. Critical and reciprocal regulation of KLF4 and SLUG in transforming growth factor beta-initiated prostate cancer epithelial-mesenchymal transition. Mol Cell Biol 2012; 32: 941-953.

3 Wang J, Place RF, Huang V, Wang X, Noonan EJ, Magyar CE et al. Prognostic value and function of KLF4 in prostate cancer: RNAa and vector-mediated overexpression identify KLF4 as an inhibitor of tumor cell growth and migration. Cancer Res 2010; 70: 10182-10191.

4 Choi BJ, Cho YG, Song JW, Kim CJ, Kim SY, Nam SW et al. Altered expression of the KLF4 in colorectal cancers. Pathol Res Pract 2006; 202: 585-589.

5 Wei D, Gong W, Kanai M, Schlunk C, Wang L, Yao JC et al. Drastic down-regulation of Kruppel-like factor 4 expression is critical in human gastric cancer development and progression. Cancer Res 2005; 65: 2746-2754.

6 Ghaleb AM, McConnell BB, Nandan MO, Katz JP, Kaestner KH, Yang VW. Haploinsufficiency of Kruppel-like factor 4 promotes adenomatous polyposis coli dependent intestinal tumorigenesis. Cancer Res 2007; 67: 7147-7154.

7 Fujimura T, Takahashi S, Urano T, Takayama K, Sugihara T, Obinata D et al. Expression of androgen and estrogen signaling components and stem cell markers to predict cancer progression and cancer-specific survival in patients with metastatic prostate cancer. Clin Cancer Res 2014; 20: 4625-4635.

8 Miyamoto DT, Zheng Y, Wittner BS, Lee RJ, Zhu H, Broderick KT et al. RNA-Seq of single prostate CTCs implicates noncanonical Wnt signaling in antiandrogen resistance. Science 2015; 349: 1351-1356.

9 Heinlein CA, Chang C. Androgen receptor in prostate cancer. Endocr Rev 2004; 25: 276-308.

10 Dong Y, Zhang H, Gao AC, Marshall JR, Ip C. Androgen receptor signaling intensity is a key factor in determining the sensitivity of prostate cancer cells to selenium inhibition of growth and cancer-specific biomarkers. Mol Cancer Ther 2005; 4: 1047-1055.

11 Aragon-Ching JB. The evolution of prostate cancer therapy: targeting the androgen receptor. Front Oncol 2014; 4: 295.

12 Culig Z. Targeting the androgen receptor in prostate cancer. Expert Opin Pharmacother 2014; 15: 1427-1437.

13 Carver BS. Strategies for targeting the androgen receptor axis in prostate cancer. Drug Discov Today 2014; 19: 1493-1497.

14 Kasinski AL, Slack FJ. Epigenetics and genetics. MicroRNAs en route to the clinic: progress in validating and targeting microRNAs for cancer therapy. Nat Rev Cancer 2011; 11: 849-864.

15 Kong YW, Ferland-McCollough D, Jackson TJ, Bushell M. microRNAs in cancer management. Lancet Oncol 2012; 13: e249-e258.

16 Hudson RS, Yi M, Esposito D, Watkins SK, Hurwitz AA, Yfantis HG et al. MicroRNA-1 is a candidate tumor suppressor and prognostic marker in human prostate cancer. Nucleic Acids Res 2012; 40: 3689-3703.

17 Martens-Uzunova ES, Jalava SE, Dits NF, van Leenders GJ, Moller S, Trapman J et al. Diagnostic and prognostic signatures from the small non-coding RNA transcriptome in prostate cancer. Oncogene 2012; 31: 978-991.

18 Ambs S, Prueitt RL, Yi M, Hudson RS, Howe TM, Petrocca F et al. Genomic profiling of microRNA and messenger RNA reveals deregulated microRNA expression in prostate cancer. Cancer Res 2008; 68: 6162-6170.

19 Liu YN, Yin JJ, Abou-Kheir W, Hynes PG, Casey OM, Fang L et al. MiR-1 and miR-200 inhibit EMT via Slug-dependent and tumorigenesis via Slug-independent mechanisms. Oncogene 2013; 32: 296-306.

20 Liu YN, Yin J, Barrett B, Sheppard-Tillman H, Li D, Casey OM et al. Loss of androgen-regulated microRNA 1 activates SRC and promotes prostate cancer bone metastasis. Mol Cell Biol 2015; 35: 1940-1951.

21 Dang DT, Chen X, Feng J, Torbenson M, Dang LH, Yang VW. Overexpression of Kruppel-like factor 4 in the human colon cancer cell line RKO leads to reduced tumorigenecity. Oncogene 2003; 22: 3424-3430.

22 Schalken J, Fitzpatrick JM. Enzalutamide: targeting the androgen signalling pathway in metastatic castration-resistant prostate cancer. BJU Int 2016; 117: 215-225.

23 Guerrero J, Alfaro IE, Gomez F, Protter AA, Bernales S. Enzalutamide, an androgen receptor signaling inhibitor, induces tumor regression in a mouse model of castration-resistant prostate cancer. Prostate 2013; 73: 1291-1305. 
24 Wang Q, Li W, Zhang Y, Yuan X, Xu K, Yu J et al. Androgen receptor regulates a distinct transcription program in androgen-independent prostate cancer. Cell 2009; 138: 245-256.

25 Cai C, He HH, Chen S, Coleman I, Wang H, Fang Z et al. Androgen receptor gene expression in prostate cancer is directly suppressed by the androgen receptor through recruitment of lysine-specific demethylase 1. Cancer Cell 2011; 20: 457-471.

26 Obinata D, Takayama K, Urano T, Murata T, Kumagai J, Fujimura T et al. Oct1 regulates cell growth of $\mathrm{LNCaP}$ cells and is a prognostic factor for prostate cancer. Int J Cancer 2012; 130: 1021-1028.

27 Nelson PS, Clegg N, Arnold H, Ferguson C, Bonham M, White J et al. The program of androgen-responsive genes in neoplastic prostate epithelium. Proc Natl Acad Sci USA 2002; 99: 11890-11895.

28 Taylor BS, Schultz N, Hieronymus H, Gopalan A, Xiao Y, Carver BS et al. Integrative genomic profiling of human prostate cancer. Cancer Cell 2010; 18: 11-22.

29 Shang Y, Myers M, Brown M. Formation of the androgen receptor transcription complex. Mol Cell 2002; 9: 601-610.

30 Wei D, Kanai M, Jia Z, Le X, Xie K. Kruppel-like factor 4 induces p27Kip1 expression in and suppresses the growth and metastasis of human pancreatic cancer cells. Cancer Res 2008; 68: 4631-4639.

31 Yori JL, Johnson E, Zhou G, Jain MK, Keri RA. Kruppel-like factor 4 inhibits epithelial-to-mesenchymal transition through regulation of E-cadherin gene expression. J Biol Chem 2010; 285: 16854-16863.

32 Yoon HS, Yang VW. Requirement of Kruppel-like factor 4 in preventing entry into mitosis following DNA damage. J Biol Chem 2004; 279: 5035-5041.

33 Chen X, Johns DC, Geiman DE, Marban E, Dang DT, Hamlin G et al. Kruppel-like factor 4 (gut-enriched Kruppel-like factor) inhibits cell proliferation by blocking G1/S progression of the cell cycle. J Biol Chem 2001; 276: 30423-30428.

34 Yoon HS, Chen X, Yang VW. Kruppel-like factor 4 mediates p53-dependent G1/S cell cycle arrest in response to DNA damage. J Biol Chem 2003; 278: 2101-2105.

35 Rowland BD, Peeper DS. KLF4, p21 and context-dependent opposing forces in cancer. Nat Rev Cancer 2006; 6: 11-23.

36 Ky N, Lim CB, Li J, Tam JP, Hamza MS, Zhao Y. KLF4 suppresses HDACi induced caspase activation and the SAPK pathway by targeting p57(Kip2). Apoptosis 2009; 14: 1095-1107.

37 Shie JL, Chen ZY, Fu M, Pestell RG, Tseng CC. Gut-enriched Kruppel-like factor represses cyclin D1 promoter activity through Sp1 motif. Nucleic Acids Res 2000; 28: 2969-2976.

38 Klaewsongkram J, Yang Y, Golech S, Katz J, Kaestner KH, Weng NP. Kruppel-like factor 4 regulates $B$ cell number and activation-induced $B$ cell proliferation. J Immunol 2007; 179: 4679-4684.
39 Shen MM, Abate-Shen C. Molecular genetics of prostate cancer: new prospects for old challenges. Genes Dev 2010; 24: 1967-2000.

40 Harris WP, Mostaghel EA, Nelson PS, Montgomery B. Androgen deprivation therapy: progress in understanding mechanisms of resistance and optimizing androgen depletion. Nat Clin Pract Urol 2009; 6: 76-85.

41 Karantanos T, Corn PG, Thompson TC. Prostate cancer progression after androgen deprivation therapy: mechanisms of castrate resistance and novel therapeutic approaches. Oncogene 2013; 32: 5501-5511.

42 Feldman BJ, Feldman D. The development of androgen-independent prostate cancer. Nat Rev Cancer 2001; 1: 34-45.

43 Niu Y, Altuwaijri S, Lai KP, Wu CT, Ricke WA, Messing EM et al. Androgen receptor is a tumor suppressor and proliferator in prostate cancer. Proc Natl Acad Sci USA 2008; 105: 12182-12187.

44 Yin J, Pollock C, Tracy K, Chock M, Martin P, Oberst M et al. Activation of the RalGEF/Ral pathway promotes prostate cancer metastasis to bone. Mol Cell Biol 2007; 27: 7538-7550

45 Yin JJ, Zhang L, Munasinghe J, Linnoila RI, Kelly K. Cediranib/AZD2171 inhibits bone and brain metastasis in a preclinical model of advanced prostate cancer. Cancer Res 2010; 70: 8662-8673.

46 Yin J, Liu YN, Tillman H, Barrett B, Hewitt S, Ylaya K et al. AR-regulated TWEAKFN14 pathway promotes prostate cancer bone metastasis. Cancer Res 2014; 74: 4306-4317.

47 Siu MK, Abou-Kheir W, Yin JJ, Chang YS, Barrett B, Suau F et al. Loss of EGFR signaling regulated miR-203 promotes prostate cancer bone metastasis and tyrosine kinase inhibitors resistance. Oncotarget 2014; 5: 3770-3784.

48 Siu MK, Tsai YC, Chang YS, Yin JJ, Suau F, Chen WY et al. Transforming growth factor-beta promotes prostate bone metastasis through induction of microRNA-96 and activation of the mTOR pathway. Oncogene 2015; 34: 4767-4776.

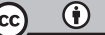

Oncogenesis is an open-access journal published by Nature Publishing Group. This work is licensed under a Creative Commons Attribution 4.0 International License. The images or other third party material in this article are included in the article's Creative Commons license, unless indicated otherwise in the credit line; if the material is not included under the Creative Commons license, users will need to obtain permission from the license holder to reproduce the material. To view a copy of this license, visit http://creativecommons.org/licenses/by/4.0/

(c) The Author(s) 2016

Supplementary Information accompanies this paper on the Oncogenesis website (http://www.nature.com/oncsis) 